\title{
Force Transducers Relative error of Zero
}

\author{
Seif M. Osman ${ }^{1}$ \\ ${ }^{1}$ National Institute of Standards (NIS) \\ Tersa St. ElHaram (Giza, Egypt) \\ Email: Seifelnasr_nis [AT] yahoo.com
}

\begin{abstract}
This study represent the influence of relative zero error on force proving instruments classification. It is directed to compare the relative zero error resulted from the three loading scenarios applied during calib ration of force proving instruments in increasing and decreasing scheme as per ISO 376:201 1. It is conclude that the maximum relative zero error resulted after the first loading series at zero position. Two methods are recommended to be proposed as a unique method to calculate the maximum relative error of zero from the first and second loading series or from the preloading series
\end{abstract}

Keywords—Force, Calibration, Relative zero error, ISO 376

\section{INTRODUCTION}

A force measurement s ystem is made up of a transducer and as sociated instrumentation. Strictly a trans ducer is a device that receives a physical stimulus (force) and changes it into another meas urable physicalquantity through a known relationship. Force transducers are classified according to the type of output signal generated to mechanical, hy d raulic, pneumatic or electric transducers [1].

Load cells are one form of electrical force trans ducers, consist of specially designed structures which perform in a predictable and repeatable manner when a force is applied. The force applied is translated into a voltage by the resistance change in strain gages which are intimately bonded to the transducer structure. The a mount of change in res is tance indicates the magnitude of deformation in the transducerstructure andhence the applied load [2]. Load cells may be characterized as transfer or working standards based on its metrological characteristic resulted from the calibration processes [3].

ISO 376:2011 fourth version [4] cancels and replaces the previous version [5]; there are two calibration schemes according to ISO 376:2011, scheme (A) with increasing and decreasing forces (Figure 1) and scheme (B) with only increasing forces (Figure 2).

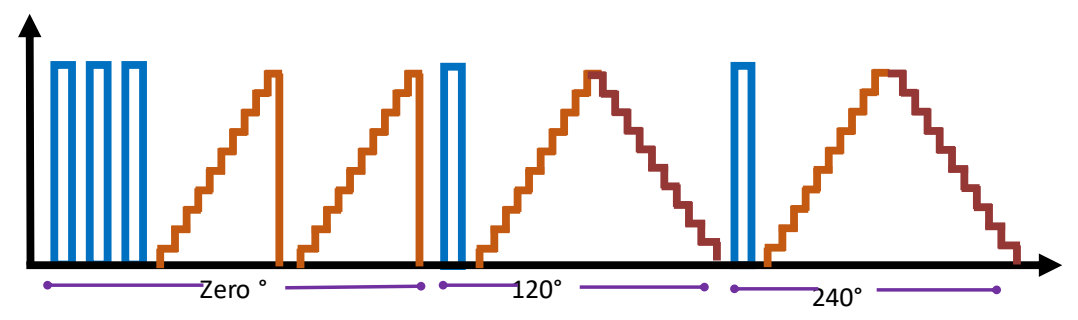

Figure 1: ISO 376 increasing \& decreasing calibration (Scheme A)

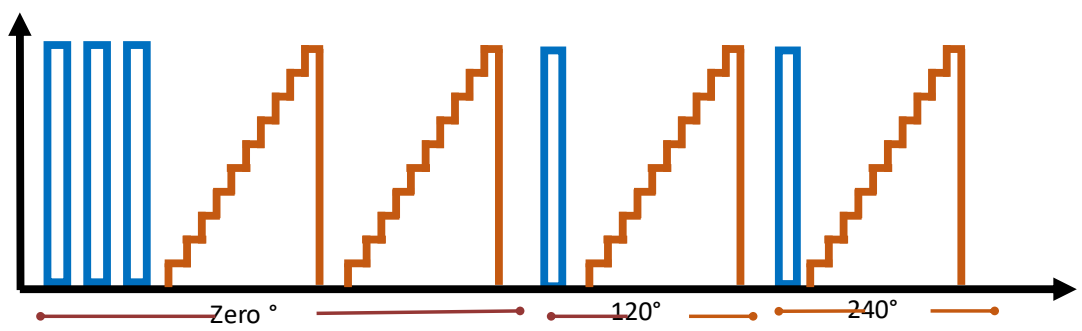

Figure 2: ISO 376 increasing calibration(Scheme B) 
ISO 376:2011 calculates and use the relative errors of Reproducibility (b), Repeatability (b`), Interpolation (fc), Zero (f0), Reversibility (v), Creep (c) to class ify the force transducers. Table (1) show limits of the relative error in class ifying force proving instruments as per ISO 376:2011 [4].

Table 1: Characteristics of the force proving instruments according to ISO376:2011

\begin{tabular}{ccccccc} 
& \multicolumn{6}{c}{ Relative e rror of the force proving ins trument \% } \\
\cline { 2 - 7 } Class & Reproducibility & Repeatability & Interpolation & zero & reversibility & Creep \\
\cline { 2 - 7 } & $\mathbf{B}$ & $\mathbf{b}$ & $\mathbf{f}_{\mathbf{c}}$ & $\mathbf{f}_{\mathbf{0}}$ & $\mathbf{V}$ & $\mathbf{c}$ \\
$\mathbf{0}$ & 0.05 & 0.025 & \pm 0.025 & \pm 0.012 & 0.07 & 0.025 \\
$\mathbf{0 . 5}$ & 0.1 & 0.05 & \pm 0.05 & \pm 0.025 & 0.15 & 0.05 \\
$\mathbf{1}$ & 0.2 & 0.1 & \pm 0.1 & \pm 0.05 & 0.3 & 0.1 \\
$\mathbf{2}$ & 0.4 & 0.2 & \pm 0.2 & \pm 0.1 & 0.5 & 0.2
\end{tabular}

2. RELATIVE ERROR OF ZERO

The relative error of zero $\left(f_{\mathrm{o}}\right)$ is an indicator on the effect of the loading process on the load cell zero output signal. According to ISO 376:2011; it is calculated from equation (1) [4].

$$
f_{0}=\frac{i_{f}-i_{0}}{X_{N}} \times 100
$$

Where:

$\mathrm{i}_{\mathrm{f}}$ is the reading on the load cell indicator after removal of force.

$\mathrm{i}_{0}$ is the reading on the load cell indicator before application of force.

$\mathrm{X}_{\mathrm{N}}$ is the res ponse corresponding to the maximum calibration force

ISO 376:2011 states that the maximum relative zero error evaluated out of thoseevaluated after the four test series should be considered in load cell classification [4]. Comparing the four test series resulted from(B) calibration scheme is mo re logic than comparing the four relative zero error resulted from scheme (A), as the relative zero error is calculated from two different loading scenarios. In the first loading scenario; the maximum force is applied gradually (set of as cen ding steps) followed by sudden load removal (one step) for two times. In the second loading scenario; themaximu m force is applied gradually (set of ascending steps) followed by gradual removal (descending steps) for two times.

\section{JUSTIFICATION AND OBJECTIVES}

The aim of this experimental inves tig ation is to compare different scenarios in calculating the relative zero error as others directed to rotation effect [6-8]. Three loading scenarios are implemented during calibration according to ISO 376:2011 (scheme A) where the maximum calibration force is applied for nine times (Figure 1). In the first loading scenario; the maximum force is applied suddenly (one step) then it is unloaded suddenly (one step) for five times. In the second loading scenario; the maximum force is applied gradually (set of ascending s teps) followed by sudden load removal(on e step) for two times. In the third loading scenario; the maximum force is applied gradually (set of ascending steps) followed by gradual removal (descending steps) for two times.

\section{EXPERIMENTAL RESULTS}

Different load cells with different capacities range from $1 \mathrm{kN}$ to $5000 \mathrm{kN}$ from five different manufact ure rs (HBM, GTM, Rever, Interface, Matest) were calibrated on a primary (Figure 3) [9] or secondary calibration machines (Figure 4) according to ISO 376:2011 scheme (A) increasing and decreasing forces. Calibration results are analyzed to classify each load cell and determine vales for the nine relative zero errors for each load cell. Table (2)represents the calib rated load cells capacity, class, the values of the nine relative errors of zero and their average value. Results reveal that transducers quite often have a problem with the zero-return after the first series, so they start with a "wrong" zero at the second series. 


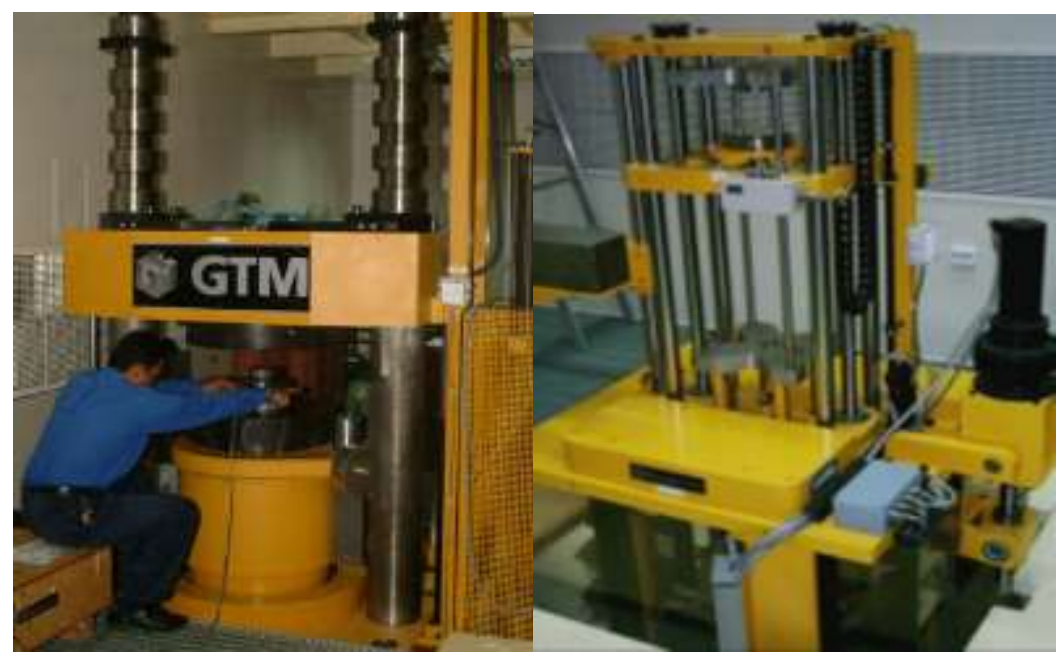

Figure 3: Example for used primary force standard machines

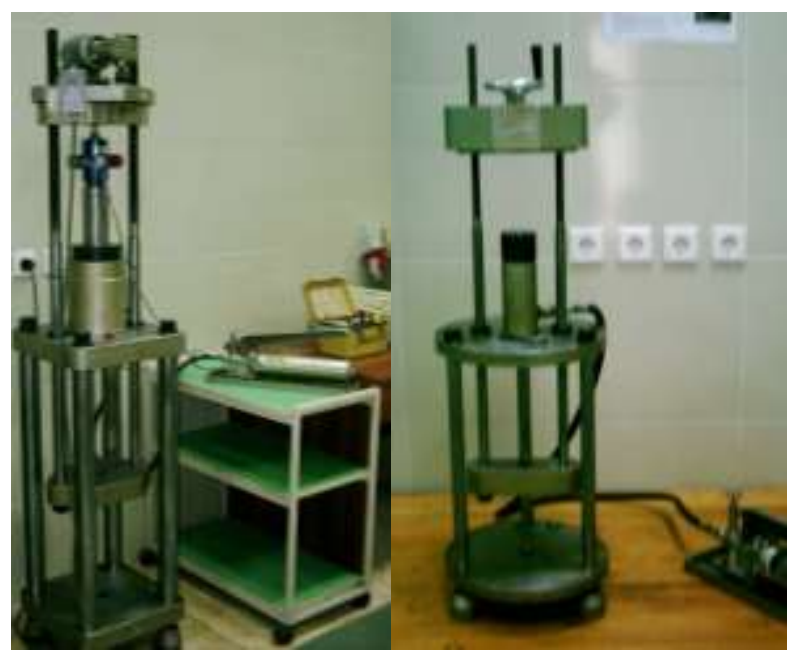

Figure 4: Example for secondary force standard machines

Table (2): Calculated relative error of zero.

\begin{tabular}{|c|c|c|c|c|c|c|c|c|c|c|c|c|}
\hline \multicolumn{3}{|c|}{ Load cell } & \multicolumn{5}{|c|}{ Preload } & \multicolumn{4}{|c|}{ Series } & \multirow{2}{*}{ Avg. } \\
\hline$\#$ & Cap. & Class & $0^{\circ}{ }_{1}$ & $\mathrm{O}_{2}$ & $0^{\circ}{ }_{3}$ & $120^{\circ}$ & $240^{\circ}$ & $\mathrm{X} 1$ & $\mathrm{X} 2$ & $\begin{array}{c}\text { X3 \& } \\
\text { X4 }\end{array}$ & $\begin{array}{c}\text { X5 \& } \\
\text { X6 }\end{array}$ & \\
\hline 1 & 1 & 0 & 0.0036 & 0.0004 & 0.0004 & 0.0005 & 0.0008 & 0.0021 & 0.0004 & 0.0003 & 0.0001 & 0.0035 \\
\hline 2 & 1 & 0 & 0.0025 & 0.0025 & 0.0025 & 0.0029 & 0.0027 & 0.0103 & 0.0076 & 0.0056 & 0.0089 & 0.0077 \\
\hline 3 & 1 & 0.5 & 0.0018 & 0.0018 & 0.0019 & 0.0014 & 0.0011 & 0.0020 & 0.0024 & 0.0026 & 0.0005 & 0.0021 \\
\hline 4 & 1 & 0 & 0.0100 & 0.0078 & 0.0079 & 0.0023 & 0.0052 & 0.0034 & 0.0098 & 0.0071 & 0.0050 & 0.0077 \\
\hline 5 & 1 & out & 0.0013 & 0.0011 & 0.0004 & 0.0008 & 0.0002 & 0.0016 & 0.0016 & 0.0005 & 0.0012 & 0.0015 \\
\hline 6 & 2 & 0 & 0.0580 & 0.0000 & 0.0000 & 0.0000 & 0.0145 & 0.0435 & 0.0290 & 0.0145 & 0.0000 & 0.0580 \\
\hline 7 & 2.5 & 0.5 & 0.0005 & 0.0010 & 0.0009 & 0.0010 & 0.0017 & 0.0029 & 0.0031 & 0.0022 & 0.0020 & 0.0027 \\
\hline 8 & 5 & 0 & 0.0007 & 0.0004 & 0.0003 & 0.0005 & 0.0004 & 0.0025 & 0.0012 & 0.0014 & 0.0007 & 0.0022 \\
\hline 9 & 5 & 0.5 & 0.0000 & 0.0000 & 0.0000 & 0.0000 & 0.0003 & 0.0038 & 0.0020 & 0.0004 & 0.0010 & 0.0038 \\
\hline 10 & 5 & 0 & 0.0135 & 0.0015 & 0.0000 & 0.0000 & 0.0005 & 0.0015 & 0.0005 & 0.0020 & 0.0010 & 0.0135 \\
\hline 11 & 5 & 0 & 0.0013 & 0.0013 & 0.0013 & 0.0007 & 0.0019 & 0.0035 & 0.0024 & 0.0012 & 0.0020 & 0.0028 \\
\hline
\end{tabular}




\begin{tabular}{|c|c|c|c|c|c|c|c|c|c|c|c|c|}
\hline \multicolumn{3}{|c|}{ Load cell } & \multicolumn{5}{|c|}{ Preload } & \multicolumn{4}{|c|}{ Series } & \multirow{2}{*}{ Avg. } \\
\hline \# & Cap. & Class & $0^{\circ}{ }_{1}$ & $0^{\circ}{ }_{2}$ & $0^{\circ}{ }_{3}$ & $120^{\circ}$ & $240^{\circ}$ & X1 & $\mathrm{X} 2$ & $\begin{array}{c}\text { X3 \& } \\
\text { X4 }\end{array}$ & $\begin{array}{c}\mathrm{X} 5 \& \\
\times 6\end{array}$ & \\
\hline 12 & 10 & 0 & 0.0010 & 0.0010 & 0.0010 & 0.0007 & 0.0006 & 0.0030 & 0.0024 & 0.0004 & 0.0004 & 0.0025 \\
\hline 13 & 10 & 0 & 0.0006 & 0.0008 & 0.0004 & 0.0001 & 0.0010 & 0.0040 & 0.0020 & 0.0000 & 0.0010 & 0.0040 \\
\hline 14 & 10 & 0 & 0.0006 & 0.0008 & 0.0004 & 0.0001 & 0.0010 & 0.0027 & 0.0020 & 0.0007 & 0.0012 & 0.0025 \\
\hline 15 & 10 & 0 & 0.0001 & 0.0008 & 0.0007 & 0.0004 & 0.0010 & 0.0048 & 0.0025 & 0.0011 & 0.0009 & 0.0046 \\
\hline 16 & 10 & 0 & 0.0017 & 0.0017 & 0.0017 & 0.0031 & 0.0030 & 0.0085 & 0.0076 & 0.0046 & 0.0045 & 0.0067 \\
\hline 17 & 10 & out & 0.0016 & 0.0014 & 0.0011 & 0.0013 & 0.0023 & 0.0045 & 0.0046 & 0.0018 & 0.0015 & 0.0035 \\
\hline 18 & 10 & out & 0.0016 & 0.0014 & 0.0011 & 0.0013 & 0.0023 & 0.0045 & 0.0046 & 0.0018 & 0.0015 & 0.0035 \\
\hline 19 & 20 & out & 0.0019 & 0.0015 & 0.0019 & 0.0015 & 0.0011 & 0.0098 & 0.0038 & 0.0034 & 0.0019 & 0.0086 \\
\hline 20 & 25 & 0.5 & 0.0000 & 0.0000 & 0.0000 & 0.0200 & 0.0400 & 0.0000 & 0.0000 & 0.0000 & 0.0000 & 0.0400 \\
\hline 21 & 25 & 1 & 0.0004 & 0.0004 & 0.0004 & 0.0005 & 0.0016 & 0.0051 & 0.0050 & 0.0006 & 0.0012 & 0.0047 \\
\hline 22 & 25 & 0.5 & 0.0006 & 0.0006 & 0.0006 & 0.0018 & 0.0012 & 0.0002 & 0.0000 & 0.0009 & 0.0007 & 0.0017 \\
\hline 23 & 25 & 0.5 & 0.0005 & 0.0016 & 0.0072 & 0.0014 & 0.0014 & 0.0053 & 0.0047 & 0.0039 & 0.0001 & 0.0071 \\
\hline 24 & 45 & 0 & 0.0016 & 0.0005 & 0.0005 & 0.0005 & 0.0010 & 0.0021 & 0.0026 & 0.0016 & 0.0068 & 0.0062 \\
\hline 25 & 45 & 2 & 0.0020 & 0.0015 & 0.0015 & 0.0015 & 0.0020 & 0.0034 & 0.0015 & 0.0113 & 0.0020 & 0.0099 \\
\hline 26 & 45 & 0 & 0.0076 & 0.0020 & 0.0026 & 0.0023 & 0.0003 & 0.0007 & 0.0010 & 0.0023 & 0.0010 & 0.0073 \\
\hline 27 & 50 & 0.5 & 0.0013 & 0.0012 & 0.0011 & 0.0019 & 0.0031 & 0.0082 & 0.0051 & 0.0036 & 0.0013 & 0.0071 \\
\hline 28 & 50 & 0 & 0.0009 & 0.0009 & 0.0009 & 0.0023 & 0.0021 & 0.0013 & 0.0011 & 0.0014 & 0.0013 & 0.0014 \\
\hline 29 & 50 & 0.5 & 0.0008 & 0.0008 & 0.0008 & 0.0014 & 0.0002 & 0.0011 & 0.0008 & 0.0011 & 0.0005 & 0.0012 \\
\hline 30 & 50 & 0 & 0.0009 & 0.0009 & 0.0009 & 0.0028 & 0.0016 & 0.0024 & 0.0015 & 0.0037 & 0.0031 & 0.0027 \\
\hline 31 & 50 & 0 & 0.0015 & 0.0015 & 0.0015 & 0.0015 & 0.0009 & 0.0001 & 0.0056 & 0.0023 & 0.0020 & 0.0055 \\
\hline 32 & 50 & 0.5 & 0.0009 & 0.0002 & 0.0012 & 0.0000 & 0.0006 & 0.0008 & 0.0010 & 0.0014 & 0.0015 & 0.0014 \\
\hline 33 & 50 & 0 & 0.0057 & 0.0028 & 0.0019 & 0.0016 & 0.0015 & 0.0013 & 0.0019 & 0.0006 & 0.0001 & 0.0056 \\
\hline 34 & 50 & 0.5 & 0.0013 & 0.0004 & 0.0004 & 0.0004 & 0.0001 & 0.0019 & 0.0010 & 0.0022 & 0.0013 & 0.0021 \\
\hline 35 & 100 & 0.5 & 0.0007 & 0.0007 & 0.0005 & 0.0007 & 0.0012 & 0.0009 & 0.0009 & 0.0008 & 0.0008 & 0.0007 \\
\hline 36 & 100 & 0 & 0.0665 & 0.0000 & 0.0000 & 0.0886 & 0.0443 & 0.0443 & 0.0443 & 0.0443 & 0.0443 & 0.0886 \\
\hline 37 & 100 & 2 & 0.0036 & 0.0004 & 0.0004 & 0.0005 & 0.0008 & 0.0021 & 0.0004 & 0.0003 & 0.0001 & 0.0035 \\
\hline 38 & 100 & 0 & 0.0066 & 0.0017 & 0.0005 & 0.0001 & 0.0005 & 0.0020 & 0.0021 & 0.0028 & 0.0013 & 0.0064 \\
\hline 39 & 100 & 0.5 & 0.0006 & 0.0006 & 0.0006 & 0.0003 & 0.0007 & 0.0013 & 0.0018 & 0.0004 & 0.0005 & 0.0015 \\
\hline 40 & 200 & 1 & 0.0005 & 0.0005 & 0.0005 & 0.0005 & 0.0004 & 0.0011 & 0.0005 & 0.0012 & 0.0011 & 0.0008 \\
\hline 41 & 200 & 0 & 0.0008 & 0.0008 & 0.0008 & 0.0008 & 0.0002 & 0.0038 & 0.0026 & 0.0006 & 0.0006 & 0.0036 \\
\hline 42 & 200 & 0 & 0.0009 & 0.0009 & 0.0009 & 0.0019 & 0.0024 & 0.0039 & 0.0021 & 0.0003 & 0.0006 & 0.0036 \\
\hline 43 & 200 & 0 & 0.0001 & 0.0000 & 0.0004 & 0.0006 & 0.0006 & 0.0019 & 0.0022 & 0.0011 & 0.0005 & 0.0022 \\
\hline 44 & 200 & 0 & 0.0000 & 0.0005 & 0.0004 & 0.0028 & 0.0004 & 0.0025 & 0.0019 & 0.0001 & 0.0011 & 0.0028 \\
\hline 45 & 200 & 1 & 0.0017 & 0.0014 & 0.0010 & 0.0005 & 0.0009 & 0.0026 & 0.0019 & 0.0005 & 0.0001 & 0.0025 \\
\hline 46 & 250 & 0.5 & 0.0021 & 0.0000 & 0.0001 & 0.0015 & 0.0016 & 0.0047 & 0.0062 & 0.0009 & 0.0016 & 0.0061 \\
\hline 47 & 250 & 0.5 & 0.0012 & 0.0012 & 0.0012 & 0.0046 & 0.0044 & 0.0041 & 0.0029 & 0.0007 & 0.0007 & 0.0039 \\
\hline 48 & 250 & 0.5 & 0.0014 & 0.0014 & 0.0014 & 0.0030 & 0.0039 & 0.0035 & 0.0035 & 0.0019 & 0.0009 & 0.0030 \\
\hline 49 & 445 & out & 0.0001 & 0.0008 & 0.0006 & 0.0005 & 0.0006 & 0.0073 & 0.0028 & 0.0008 & 0.0019 & 0.0072 \\
\hline 50 & 500 & 0.5 & 0.0034 & 0.0034 & 0.0034 & 0.0004 & 0.0017 & 0.0045 & 0.0040 & 0.0031 & 0.0039 & 0.0041 \\
\hline 51 & 500 & 0.5 & 0.3501 & 0.0499 & 0.0333 & 0.0331 & 0.0831 & 0.0166 & 0.0498 & 0.0000 & 0.0166 & 0.3501 \\
\hline 52 & 500 & out & 0.0015 & 0.0015 & 0.0016 & 0.0009 & 0.0004 & 0.0039 & 0.0043 & 0.0011 & 0.0006 & 0.0039 \\
\hline
\end{tabular}




\begin{tabular}{|c|c|c|c|c|c|c|c|c|c|c|c|c|}
\hline \multicolumn{3}{|c|}{ Load cell } & \multicolumn{5}{|c|}{ Preload } & \multicolumn{4}{|c|}{ Series } & \multirow{2}{*}{ Avg. } \\
\hline$\#$ & Cap. & Class & $0^{\circ}{ }_{1}$ & $\mathrm{O}^{\circ}{ }_{2}$ & $0^{\circ}{ }_{3}$ & $120^{\circ}$ & $240^{\circ}$ & X1 & X2 & $\begin{array}{c}X 3 \& \\
X 4\end{array}$ & $\begin{array}{c}\text { X5 \& } \\
\times 6\end{array}$ & \\
\hline 53 & 500 & 0 & 0.0015 & 0.0018 & 0.0013 & 0.0001 & 0.0013 & 0.0045 & 0.0042 & 0.0011 & 0.0012 & 0.0043 \\
\hline 54 & 500 & 0 & 0.0002 & 0.0002 & 0.0002 & 0.0021 & 0.0036 & 0.0026 & 0.0037 & 0.0021 & 0.0034 & 0.0035 \\
\hline 55 & 1000 & 0 & 0.0002 & 0.0002 & 0.0002 & 0.0039 & 0.0083 & 0.0008 & 0.0002 & 0.0086 & 0.0044 & 0.0084 \\
\hline 56 & 1000 & 0 & 0.0009 & 0.0009 & 0.0009 & 0.0008 & 0.0034 & 0.0014 & 0.0003 & 0.0082 & 0.0104 & 0.0101 \\
\hline 57 & 1000 & 0 & 0.0001 & 0.0001 & 0.0001 & 0.0008 & 0.0016 & 0.0014 & 0.0012 & 0.0061 & 0.0076 & 0.0075 \\
\hline 58 & 1000 & 0 & 0.0018 & 0.0000 & 0.0001 & 0.0005 & 0.0021 & 0.0040 & 0.0023 & 0.0044 & 0.0037 & 0.0044 \\
\hline 59 & 1000 & 0 & 0.0023 & 0.0002 & 0.0011 & 0.0057 & 0.0060 & 0.0110 & 0.0109 & 0.0021 & 0.0026 & 0.0108 \\
\hline 60 & 1000 & 0 & 0.0000 & 0.0005 & 0.0004 & 0.0028 & 0.0004 & 0.0111 & 0.0103 & 0.0029 & 0.0022 & 0.0111 \\
\hline 61 & 1200 & 2 & 0.0019 & 0.0019 & 0.0019 & 0.0038 & 0.0038 & 0.0053 & 0.0047 & 0.0085 & 0.0014 & 0.0071 \\
\hline 62 & 1200 & 2 & 0.0023 & 0.0023 & 0.0023 & 0.0023 & 0.0028 & 0.0053 & 0.0050 & 0.0008 & 0.0014 & 0.0046 \\
\hline 63 & 2000 & Out & 0.0617 & 0.1387 & 0.0154 & 0.0616 & 0.3082 & 0.0000 & 0.0154 & 0.0462 & 0.0154 & 0.3082 \\
\hline 64 & 3000 & 0.5 & 0.0062 & 0.0009 & 0.0009 & 0.0019 & 0.0019 & 0.0028 & 0.0033 & 0.0005 & 0.0033 & 0.0057 \\
\hline 65 & 3000 & 0 & 0.0028 & 0.0012 & 0.0005 & 0.0009 & 0.0009 & 0.0044 & 0.0000 & 0.0074 & 0.0038 & 0.0074 \\
\hline 66 & 3000 & 1 & 0.0065 & 0.0000 & 0.0065 & 0.0130 & 0.0000 & 0.0000 & 0.0000 & 0.0065 & 0.0000 & 0.0130 \\
\hline 67 & 3000 & Out & 0.0000 & 0.0154 & 0.0154 & 0.0922 & 0.1076 & 0.0462 & 0.0615 & 0.0154 & 0.0308 & 0.1076 \\
\hline 68 & 5000 & 0 & 0.0016 & 0.0016 & 0.0016 & 0.0009 & 0.0008 & 0.0046 & 0.0028 & 0.0004 & 0.0020 & 0.0042 \\
\hline 69 & 5000 & 1 & 0.0053 & 0.0053 & 0.0053 & 0.0119 & 0.0079 & 0.0188 & 0.0149 & 0.0035 & 0.0017 & 0.0171 \\
\hline 70 & 5000 & 0 & 0.0019 & 0.0019 & 0.0019 & 0.0009 & 0.0015 & 0.0055 & 0.0033 & 0.0002 & 0.0014 & 0.0053 \\
\hline
\end{tabular}

\section{RESULTS ANALYSIS}

Figure (5) shows the distribution of the maximum relative zero error of the seventy load cells. The maximum $\left(f_{\mathrm{o}}\right)$ was recorded 25 times after the $1^{\text {st }}$ loading series at zero position, 10 times after the $1^{\text {st }}$ preloading series at zero position, 9 times after the $2^{\text {nd }}$ loading series at zero position and after the $3^{\text {rd }} \& 4^{\text {th }}$ loading $\&$ unloading series, 7 times after preloading series at $120^{\circ}, 5$ times after preloading series at $240^{\circ}$, one time 10 times after the $3^{\text {rd }}$ preloading series at ze ro position and was not recorded after $2^{\text {nd }}$ preloading series at zero position.

Results reveal that transducers quite often have a problem with the zero-return after the first series, so the y s tart with a "wrong" zero at the second series in a phenomena known by "Late zero effect".

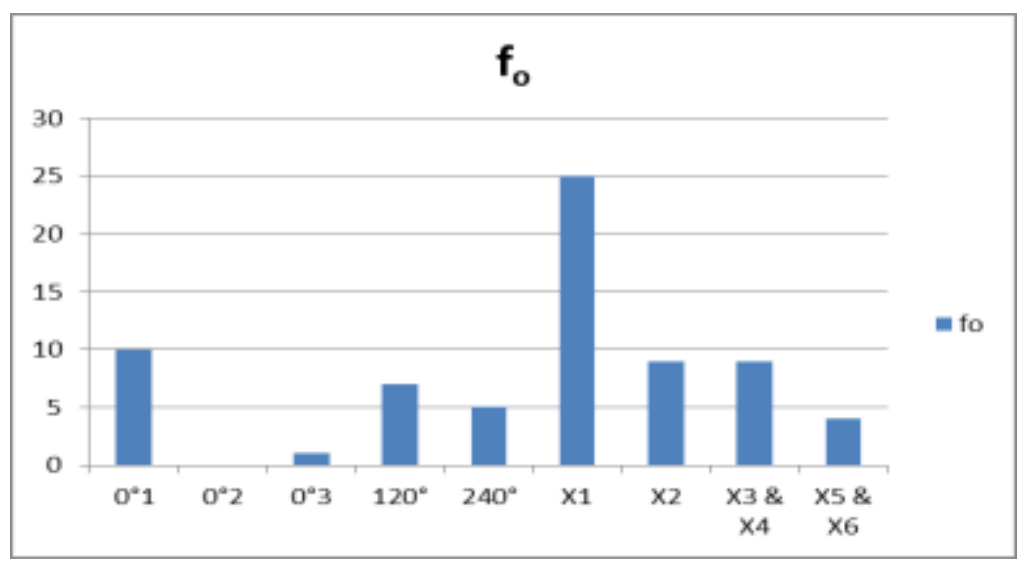

Figure 5: Maximum relative error of zero distributions

To unify a base to calculate the relative error of zero in force proving instruments calibration, five approaches for calculating the relative error of zero are proposed and compared to each other. Table (3) shows the five approach es for the relative error of zero calculations. 
Table (3). Proposed groups to unify relative error of zero calculation.

\begin{tabular}{|c|l|}
\hline Group & \multicolumn{1}{|c|}{ Des cription } \\
\hline M & The maximum $\left(f_{0}\right)$ evaluated fromthe $1^{\text {st }}$ and $2^{\text {nd }}$ loading series. \\
\hline $\mathrm{N}$ & The maximum $\left(f_{\mathrm{o}}\right)$ evaluated fromthe $3^{\text {rd }}, 4^{\text {th }}$ and $5^{\text {th }}$ preload. \\
\hline O & The maximum $\left(f_{0}\right)$ evaluated fromthe $1^{\text {st }}$ and $2^{\text {nd }}$ preload. \\
\hline P & The maximum $\left(f_{0}\right)$ evaluated fromthe $1^{\text {st }}, 2^{\text {nd }}, 3^{\text {rd }}, 4^{\text {th }}$ and $5^{\text {th }}$ preload. \\
\hline Q & The maximum $\left(f_{0}\right)$ evaluated fromthe $\left(3^{\text {rd }}, 4^{\text {th }}\right)$ and $\left(5^{\text {th }}, 6^{\text {th }}\right)$ loading $\&$ unloading. \\
\hline
\end{tabular}

Regrouping the results in Table (2) as per the proposed approaches listed in Table (3) shows that; Group (M) is more representative than the other group as it repres ents $48.6 \%$ of the maximum relative error of zero while group (O) is the less representative. Table (4) represents the probability of occurrence of maximum relative error of zero and Fig u re (6) represents the number of occurrence of maximum relative error of zero out of the seventy load cells

Table 4: Probability of maximum relative error of zero occurrences.

\begin{tabular}{|c|c|}
\hline Group & Probability of max. $\boldsymbol{f}_{\mathbf{o}}$ \\
\hline M & $48.6 \%$ \\
\hline N & $18.6 \%$ \\
\hline O & $14.3 \%$ \\
\hline P & $32.9 \%$ \\
\hline Q & $18.6 \%$ \\
\hline
\end{tabular}

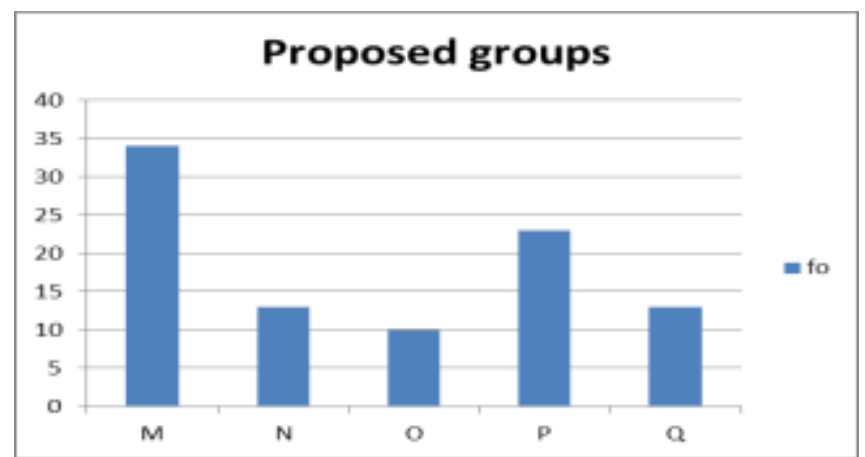

Figure (6): Maximum relative error of zero according to the proposed groups

Based on the results and analysis it is proposed to calculate the maximum relative error of zero from the $1^{\text {st }}$ and $2^{\text {nd }}$ loading series ( Group M) or from the $1^{\text {st }}, 2^{\text {nd }}, 3^{\text {rd }}, 4^{\text {th }}$ and $5^{\text {th }}$ preload ( Group P) and to indicate in the calibration certificate the method used to calculate the relative error of zero.

\section{CONCLUSION}

As a result of evaluating the results it is concluded that the maximum relative zero error was after the first loading series at zero position, this problem with the zero-return after the first series, lead to start with a "wrong " zero at the sec ond series, increasing the time between the end of the first loading series and the start of the second loading series may help to reduce the late zero effect.

Two methods are recommended to and proposed as a unique method to calculate the maximu m relative error of zero from the first and second loading series or from the preloading series, the selected me thod must be documented and certified in the calibration certificate as same as stating the relative creeperror calculation method. 


\section{REFERENCES}

[1] Seif. M. Osman, Ebtisam H. Hasan, H. M. El-Hakeem, R. M. Rashad, F. Kouta "Conceptual Design of MultiCapacity Load Cell" Proceedings of $16^{\text {th }}$ International Congress of Metrology 7-10 October 2013 Paris, France. DOI:10.1051/metrology/201203002

[2] Dan MihaiStefanescu, Alexandru Stefanescu."Criteria for Choosing theElastic Elements of Force Transducers" Proceedings of the 17th International conference IMEKO, pp. 134-140, Istanbul, Turkey, Sept.2006.

[3] Seif. M. Osman, R.Kumme, H. M. El-Hakeem, F. Löffler, Ebtisam H. Hasan, R.M.Rashad, F. Kouta "Force Transducer with Different Capacities" Proceedings of XXI IMEKO World Congress Aug. 30 - Sept. 4, 2015, Prague, Czech Republic

[4] ISO 376:2011; "Metallic materials-Calibration of force proving instruments used for the verification of uniaxial testing machines".

https://www.iso.org/standard/44661.html

[5] ISO 376:2004; "Metallic materials-Calibration of force proving instruments used for the verification of uniaxial testing machines".

https://www.iso.org/standard/44661.html

[6] Wei Liang, Xiao-xiang Yang, JinhuiYao "Rotation effects of force transducer on the output of the build -up system", Meas urement, Volume 138, May 2019, Pages 659-671,https://doi.org/10.1016/j.measurement.2019.01.071

[7] Dae-ImKang, Chang-SunHong "Rotation effects of force transducers on the output of a build-up system" Measurement, Volume 14, Issue 2, December 1994, Pages 147-156, https://doi.org/10.1016/0263-2241(94)90023-X

[8] Tieping Wei, Xiaoxiang Yang, JinhuiYao, Hang Xu, "The influence of a balanced structure on the rotation effect of a build-up system" Measurement, Volume 61, February 2015, Pages 162-168, https://doi.org/10.1016/j.measurement.2014.10.043

[9] El-sayed A. A., El-Hakeem H.M., Gloeckner B., Allgeier T. Performance Evaluation and Metrological Characteristics of a dead weight force Stand ard Machine with substitute load control system. Proceedings of IMEKO conference, 2002 